\title{
Cardioprotective activity of placental growth factor in a rat model of acute myocardial infarction: nanoparticle-based delivery versus direct myocardial injection
}

Zhe-xin Lu', Li-li Mao ${ }^{2}$, Feng Lian ${ }^{1 *}$, Jun He ${ }^{2^{*}}$, Wen-tian Zhang ${ }^{1}$, Chen-yang Dai ${ }^{1}$, Song Xue ${ }^{1}$, Wei-gen Lu ${ }^{2}$ and Hong-sheng Zhu'

\begin{abstract}
Background: To comparatively evaluate the cardioprotective activity of placental growth factor (PGF) delivered through direct injection and a nanoparticle-based system respectively and to study the underlying mechanisms in a rat model of acute myocardial infarction (AMI).

Methods: Poly lactic-Co-glycolic acid (PLGA)-based PGF-carrying nanoparticles (PGF-PLGA $A_{N P S}$ ) were created. The mean size and morphology of particles were analyzed with particle size analyzer and transmission electronic microscopy (TEM). Encapsulation efficiency and sustained-release dose curve were analyzed by ELISA. Sprague-Dawley rats were randomized into four groups $(n=10)$. While animals in the first group were left untreated as controls, those in the other 3 groups underwent surgical induction of AMl, followed by treatment with physiological saline, PGF, and PGF-PLGA $\mathrm{NPs}$ respectively. Cardiac function was evaluated by transthoracic echocardiography at 4 weeks after treatment. At 6 weeks, rats were sacrificed, infarction size was analyzed with Masson trichrome staining, and protein contents of TIMP-2, MT1-MMP and MMP-2 at the infarction border were determined by immunohistochemistry and western blotting analysis.

Results: PGF was released for at least 15 days, showing successful preparation of PGF-PLGA $A_{N P s}$. Coronary artery ligation successfully induced AMI. Compared to physiological saline control, PGF, injected to the myocardium either as a nude molecule or in a form of nanoparticles, significantly reduced infarction size, improved cardiac function, and elevated myocardial expression of TIMP-2, MT1-MMP, and MMP-2 $(P<0.05)$. The effect of PGF-PLGA NPs $_{\text {sas }}$ more pronounced than that of non-encapsulated PGF $(P<0.05)$.

Conclusion: Target PGF delivery to myocardium may improve cardiac function after AMI in rats. PLGA-based nanoparticles appear to be a better approach to delivery PGF. PGF exerts its cardioprotective effect at least partially through regulating metalloproteinase-mediated myocardial tissue remodeling.
\end{abstract}

Keywords: Placental growth factor, Acute myocardial infarction, Cardiac function, Nanoparticles-mediated drug delivery, Vicious ventricular remodeling

\footnotetext{
* Correspondence: dr.lianfeng@hotmail.com; chinaynhe@163.com

'Department of Cardiovascular Surgery, Ren Ji Hospital, School of Medicine, Shanghai Jiao Tong University, 160 Pu-Jian Rd, Shanghai 200127, People's Republic of China

${ }^{2}$ National Pharmaceutical Engineering Research Center, China State Institute of Pharmaceutical Industry, 1111 Zhong-shan North 1 Rd, Shanghai 200437, People's Republic of China
}

\section{Biomed Central}

(c) 2014 Lu et al.; licensee BioMed Central Ltd. This is an Open Access article distributed under the terms of the Creative Commons Attribution License (http://creativecommons.org/licenses/by/2.0), which permits unrestricted use, distribution, and reproduction in any medium, provided the original work is properly credited. The Creative Commons Public Domain Dedication waiver (http://creativecommons.org/publicdomain/zero/1.0/) applies to the data made available in this article unless otherwise stated. 


\section{Background}

Paracrine factors derived from stem cells have been demonstrated to be capable of improving cardiac function after myocardial infarction (MI) and are now becoming a hot topic of myocardial regeneration research $[1,2]$. One of these factors is placental growth factor (PGF). A beneficial role for PGF in heart failure has been previously demonstrated in an animal study where autoimplantation of myoblasts overexpressing PGF into myocardium resulted in a significant functional improvement in the failing heart after ischemic MI [3]. However, the mechanisms underlying the benefits of PGF in MI-associated heart failure have yet to be further elucidated.

It has been documented that MI results in remodeling of the myocardium, including the extracellular matrix (ECM) [4]. Tissue inhibitors of metalloproteinases (TIMPs) are critical regulators of ECM integrity, capable of inhibiting the activity of ECM-degrading enzymes, matrix metalloproteinases (MMPs) [5]. Numerous types of MMPs have been identified in mammals, of which membranetype-I MMP (MT1-MMP) is of a particular importance in the regulation of ECM integrity. Through activating MMP-2, MT1-MMP may aggravate the degradation and loss of ECM [6]. However, it remains unclear whether PGF may exerts its beneficial effect against ischemic myocardial injury through regulating MMP-dependent ECM degradation in the myocardium.

Various approaches to delivery exogenous agents including PGF have been developed, of which, nanoparticles have attracted considerable attention in recent years worldwide [7]. Poly (lactic-co-glycolic acid) (PLGA), a biodegradable polymer approved by U.S. Food and Drug Administration for drug delivery, is one of the widely used types of carriers [8]. Due to its properties of surface erosion and subsequent constant matrix erosion, PLGA allows for slow drug release [9]. Besides sustained release, PLGA-based nanoparticles as drug carriers possess advantages of increased freedom and better permeability into interstitial spaces. Nevertheless, delivery of PGFcarrying PLGA nanoparticles (PGF-PLGA $\mathrm{NPs}_{\mathrm{s}}$ ) into myocardium has been seldom reported.

This study aimed to investigate the therapeutic efficacy of PGF-PLGA $\mathrm{NPs}_{\mathrm{N}}$-bearing nanoparticles against acute myocardial infarction (AMI) in comparison with direct administration of PGF as well as the mechanistic role of TIMP-2, MT1-MMP and MMP-2-regulated cardiac remodeling in PGF-mediated cardioprotection in a rat model.

\section{Methods}

All human studies have been approved by shanghai Ren Ji Hospital Ethics Committee and performed in accordance with the ethical standards

\section{Preparation of PGF-PLGA $\mathrm{NPs}_{\mathrm{N}}$}

PLGA (100 mg; block ratio: 50:50; viscosity: $0.53 \mathrm{dl} / \mathrm{g}$; average molecular weight: $6.0 \times 10^{4}$ ), purchased from Shandong Institute of Medical Devices (Shandong, China), was dissolved in $0.5 \mathrm{ml}$ ethyl acetate as an oil phase $(\mathrm{O})$. Recombinant human PGF, purchased from R\&D Systems (catalog \#264-PG-050, Minneapolis, MN, USA), was dissolved in $\mathrm{H}_{2} \mathrm{O}(0.50 \mu \mathrm{g} / \mu \mathrm{l})$ as a water phase (W1). W1 was then dripped slowly into $\mathrm{O}$ to create the W1/O emulsion through ultrasonication. W1/O was added into $2 \mathrm{ml}$ $19 \%$ pluronic F-68 solution to form W1/O/W2 double emulsions. Ethyl acetate was evaporated under a vacuum and the PLGA nanoparticles were collected after high speed centrifugation (6000 rpm, $8 \mathrm{~min}$ ). Finally, PGFPLGA $_{N P s}$ were lyophilized. The dose of PGF was chosen based on a previous study [10].

\section{Assessment of in vitro release kinetics and encapsulation efficiency}

To analyze in vitro release kinetics, PGF-PLGA $A_{N P s}$ (containing $30 \mu \mathrm{g}$ PGF) were suspended in $16 \mathrm{ml}$ of phosphate-buffered saline (PBS, PH 7.4). The suspension solution was then placed in an environment of $37^{\circ} \mathrm{C}$ with horizontal shaking for 15 days. During this period, $1 \mathrm{ml}$ of the medium was sampled twice (12 $\mathrm{h}$ and $24 \mathrm{~h}$ ) on the first day and once every two days thereafter. The concentration of PGF released at different time points was determined by enzyme-linked immunosorbent assay (ELISA) as previously described [11]. All experiments were conducted in triplicate. The amount of encapsulated PGF was determined as the difference between the initial amount and residual amount in the supernatant. The average size and morphology of the nanoparticles were assessed under the NICOMP380 ZLS (Particle sizing systems, USA) and H-600 transmission electron microscopy (HITACHI, Japan). The percent encapsulation efficiency was calculated as: $=\frac{\text { (Mass of total drug-Mass of free drug) }}{\text { Mass of total drug }} \times 100 \%$, and the drug loading as: $=\frac{(\text { Mass of total drug-Mass of free drug) }}{\text { Mass of microspheres }} \times 100 \%$.

\section{Animals, AMI induction and treatment}

Sprague-Dawley rats $(250 \pm 50$ g, 8-10 weeks) were obtained from the Experimental Animal Center, Shanghai Jiaotong University-Affiliated Renji Hospital, China. They were housed in groups (4 per cage) with free access to a regular diet and clean drinking water. All the experimental procedures described below adhered strictly to the guidelines set forth by the National Science and Technology Commission of China and approved by the institutional ethics committee.

For AMI induction, rats were anesthetized with an intraperitoneal dose of $0.2 \mathrm{ml} 2 \%$ pentobarbital under intubated mechanical ventilation at 80 breaths per minute. Thoracotomy was performed through the fourth intercostal space, 
and the left anterior descending coronary artery was identified and ligated with 6.0 prolene suture in the middle portion. A few minutes after ligation, pallor and akinesia were seen in the anterior wall and apical left ventricular area. The interface between the pale and normal areas was defined as 'infarction border zone'. The presence of infarction zone and ST segment elevation were considered as criteria of successful induction of AMI. A group of animals $(n=10)$ were left untreated controls. The animals that were successfully induced to develop AMI were randomized to receive $30 \mu \mathrm{lBS}(\mathrm{n}=10)$, non-encapsulated PGF $(2 \mu \mathrm{g}$ in $30 \mu \mathrm{l}$ solution, $\mathrm{n}=10)$, and PGF-PLGA $\mathrm{NPs}_{\mathrm{s}}(2 \mu \mathrm{g}$ in $30 \mu \mathrm{l}$ solution, $\mathrm{n}=10$ ), respectively. The injection of $30 \mu \mathrm{l}$ was administrated around the pale areas: upper, center, and lower with $10 \mu \mathrm{l}$ in each area. All agents were delivered to the infarction border zone through intramyocardial injection using a micro-syringe.

\section{Cardiac function determination}

Echocardiographic examinations were performed 4 weeks after surgery under inhaled isoflurane $(0.2 \%)$ anesthesia, with the Vevo770 ultrasound system (Visualsonics Inc., Toronto, Canada). The frequency of the probe was set at $17.5 \mathrm{MHz}$, sampling frequency in M-mode at $1000 / \mathrm{s}$, and scanning speed $50-100 \mathrm{~mm} / \mathrm{s}$. The probes were placed on precordium and the detection was carried out from the section of ventricular bands. Left ventricular anterior wall (LVAW) thickness, left ventricular end-diastolic diameters (LVEDD), and left ventricular end-systolic diameters (LVESD) were measured. Based on these measurements, left ventricular end-diastolic volume (LVEDV), left ventricular endsystolic volume (LVESV), left ventricular ejection fraction (LVEF) and left ventricular fraction shortening (LVFS) were calculated as follows:

$$
\begin{aligned}
& \text { LVEDV }=\frac{7.0 \times \text { LVEDD }^{3}}{(2.4+\text { LVEDD }) ;^{3}} \\
& \text { LVESV }=\frac{7.0 \times \text { LVESD }^{3}}{(2.4+\text { LVESD }) ;} \\
& \text { LVEF }=\frac{(\text { LVEDV-LVESV })}{\text { LVEDV }} \times 100 \% ; \\
& \text { LVFS }=\frac{(\text { LVEDD }- \text { LVESD })}{\text { LVEDD }} \times 100 \% .
\end{aligned}
$$

\section{Scar area analysis}

Six weeks after AMI surgery, rats were anesthetized by inhaled isoflurane $(0.2 \%)$ followed by cardiac perfusion with $100 \mathrm{ml} \mathrm{4 \%}$ triformol. Animals were then sacrificed by rapid excision of heart. Hearts were excised and soaked immediately in saline to remove the excess blood from the ventricles before being fixed in $4 \%$ triformol. Paraffin- embedded samples were sectioned at $5 \mu \mathrm{m}$ and Masson's trichrome staining was performed. Digital images of the stained sections were captured and tissue scar was analyzed with Image Pro-Plus 6.0 software. The size of infarct was also calculated as previously described [12].

\section{Imunohistochemical analysis}

This was performed using the DAKO EnVision ${ }^{\text {Tax }}+$ System $^{2}$ (Glostrup, Denmark). Briefly, paraffin-embedded sections $(5 \mu \mathrm{m})$ were dewaxed, and incubated with the primary antibody TIMP-2 (Abcam, Cambridge, MA, USA, catalog \#ab1828), MT1-MMP (Abcam, catalog \#ab51074), MMP-2 (Abcam, catalog \# ab80737) and Envision. After the visualization with diaminobenzidine (DAB), images were acquired under the Axioplan 2 automatic fluorescence microscopy (Carl Zeiss) and the intensity of the corresponding protein bands were analyzed using the Axioplan 2 imaging software.

\section{Western blotting analysis}

Infarcted tissue specimens collected from the border zone were homogenized in $1 \%$ Triton X-100, $10 \mathrm{mM}$ Tris- $\mathrm{HCl}$ (PH 7.6). After centrifugation at $\mathrm{xxxx} g$ for $\mathrm{xx}$ min, the supernatant was collected. Concentration of total proteins in the supernatant was quantitated. Ten $\mu \mathrm{g}$ proteins from each sample prepared in a buffer containing $2 \%$ sodium dodecyl sulfate (SDS) were separated by electrophoresis on a SDS-PAGE and then transferred to a nitrocellulose membrane blot. After blocking in 5\% skimmed milk in TBST (0.1\% Tween 20) for 1 hour, the blot was incubated with primary antibodies respectively against TIMP-2 (Abcam, ab1828), MT1-MMP (Abcam, ab51074), MMP-2 (Abcam, ab80737) at $4^{\circ} \mathrm{C}$ overnight. Following washing, the blot was incubated with a HRP anti-rabbit secondary antibody (Santa Crutz, CA, USA) for 1 hour at room temperature. After another three rounds (10 min/time) of washing in TBST, the blot was developed by enhanced chemiluminescence (ECL) as instructed by the manufacturer. For the purpose of loading control, the blot was stripped and re-probed with GAPDH. The immunoreactive bands of the interest protein and GAPDH protein were densitometricaly analyzed with Image J software (National Institutes of Health, USA).

\section{Statistical analysis}

Data were expressed as mean \pm standard deviation. Intergroup comparison was analyzed by $\mathrm{t}$ test, and the intra comparison was conducted by paired $t$ test. All statistical analyses were performed using SPSS 10.7 software (Chicago, IL, USA). Differences were considered significant when $\mathrm{P}<0.05$. 


\section{Results}

Characterization of PGF-PLGA NPs $_{\text {s }}$

PGF-PGF-PLGA $A_{\mathrm{NPs}}$ had a regular spherical shape with good integrity, even distribution, and smooth surface, as demonstrated by electron microscopy (Figure 1A). The average diameter of the nanoparticles was $233.9 \pm 15.3 \mathrm{~nm}$ and the average encapsulation efficiency was $43.52 \pm$ $4.21 \%$ (Figure 1B).

\section{In vitro release kinetics of PGF-PLGA}

Shown in Figure 2 are concentrations of PGF released from PGF-PLGA $\mathrm{NPs}_{\mathrm{N}}$ at different time points during the 15-day experiment as determined by ELISA. In the first 1-5 days, PGF release was rapid, amounting for more than $60 \%$ of the total release. After that, the daily release amount became more stable. By day 14 or $15,80 \%$ of PGF was released.

\section{Echocardiography findings}

Echocardiography was performed on all animals at 4 weeks after surgery. Compared to animals in the sham group (Figure 3A), those in the other three groups showed different degrees of enhanced myocardial echo, thinning of the left ventricular wall and weakened contractions (Figure 3B-D). The LVEF, LVFS, and LVAW in PGF$\mathrm{PLGA}_{\mathrm{NPs}}$ group were all significantly higher than those in the PGF and AMI control groups $(\mathrm{P}<0.05$, Table 1$)$.

\section{Myocardial scar}

Shown in Figure 4 are Masson trichrome staining results. There were significant differences $(\mathrm{P}<0.05)$ in the average scar area between groups (Figure 4A). In the cardiac tissue sections, viable myocardium was stained red, while fibrosis due to infarction damage was stained blue. In the sham operation control group, no area was stained blue (Figure 4B1). In contrast, the infarcted zone in the non-treatment AMI control group was filled with blue scar tissues (Figure 4B2). Treatment with both non-encapsulated PGF (Figure 4B3) and

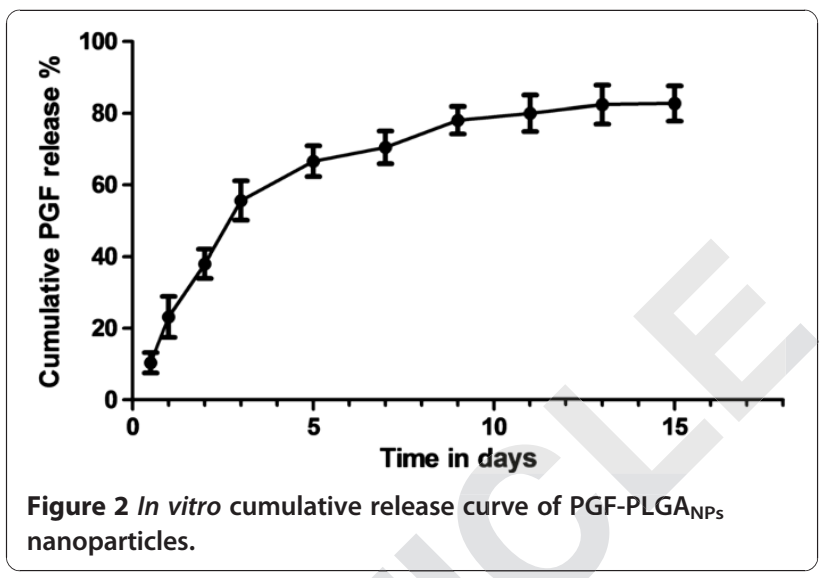

PGF-PLGA $A_{\text {NPs }}$ (Figure 4B4) reduced the area stained blue and the intensity of blue staining. However, the ef-

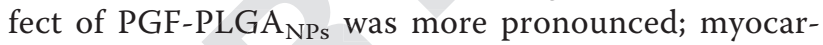
dial compensatory hypertrophy was seen in the PGF group.

\section{Protein contents of TIMP-2, MT1-MMP, and MMP-2}

Shown in Figure 5 are the results of immunohistochemical staining assessment of TIMP-2, MT1-MMP, and MMP-2 protein signals in the cardiac specimens (Figure 5). The immunoreactive signal for TIMP-2 was strong in the sham operation group but became significantly weakened after AMI surgery $(P<0.05)$. Both nonencapsulated PGF and PGF-PLGA $\mathrm{NPs}_{\mathrm{N}}$ significantly attenuated $\mathrm{AMI}$-associated decrease in the reactive signal for TIMP-2 $(\mathrm{P}<0.05)$. In contrast, immunoreactive signals for MT1-MMP and MMP-2 all showed a pattern of changes in different groups totally opposite to the pattern for TIMP-2.

Changes in TIMP-2, MT1-MMP, and MMP-2 protein contents in different groups were also analyzed by Western blotting. Similar patterns to those for these proteins shown in immunohistochemical staining were observed (Figure 6).
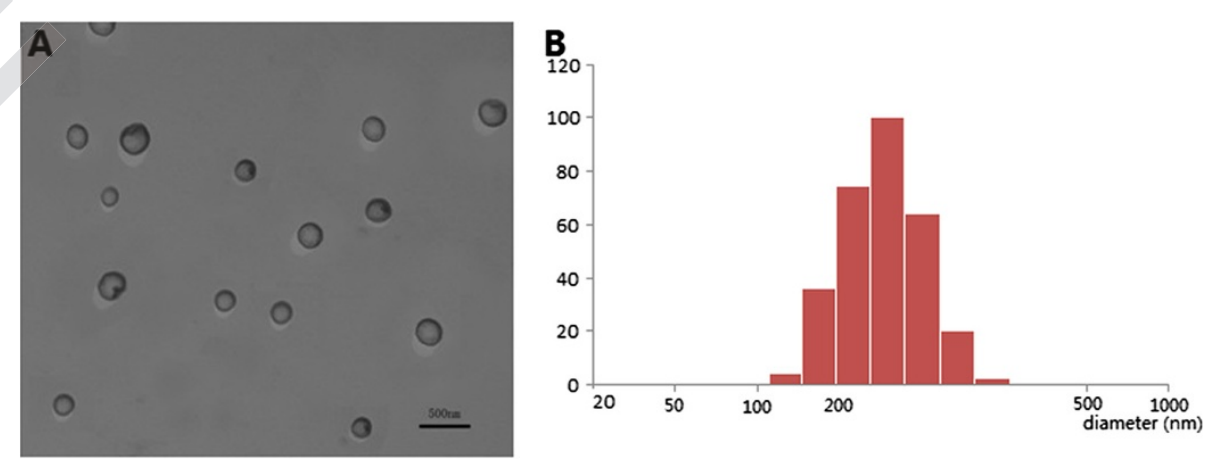

Figure 1 Characterization of nanoparticles. A: morphology revealed by transmission microscopy. B: bar graphs, showing the average diameters detected by NICOMP 380 ZLS. 

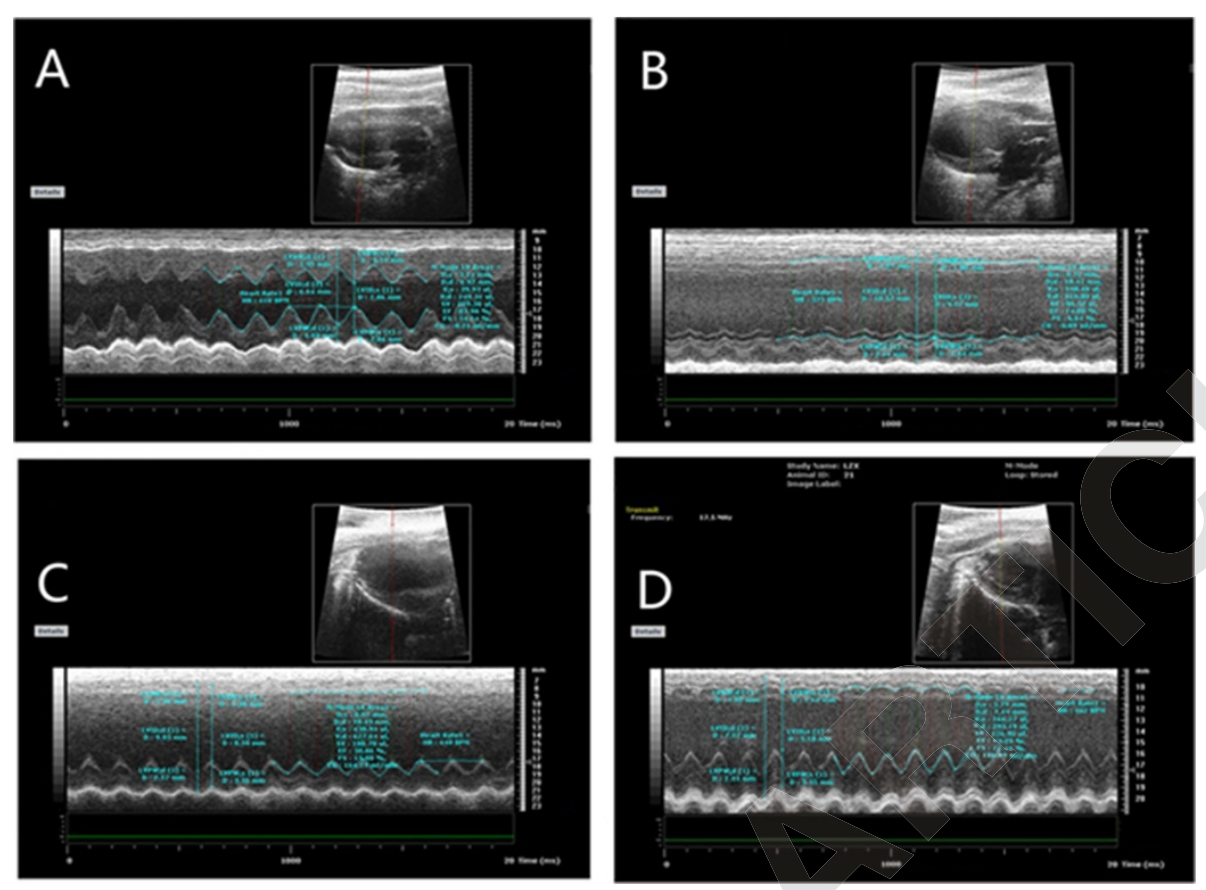

Figure 3 Echocardiography of rats in the control group (A), AMI group (B), PGF group (C) and PGF-PLGA $\mathrm{NPs}_{\text {group }}(\mathrm{D})$ at the end of the fourth week after AMI surgery.

\section{Discussion}

MI is a life-threatening clinical condition. Even though the symptoms of myocardial ischemia can be partly improved by pharmacologic agents, delivered either surgically or non-surgically, yet the malignant myocardial remodeling caused by MI cannot be reversed. PGF, one of the cytokines belonging to VEGF family, binds to flt-1 receptors in its target cells including cardiac myocytes, cardiac fibroblasts, and bone marrow derived cells [13] and then activates downstream signals important in myocardial damage repair [14,15], angiogenesis [16], inflammatory response [17], and peripheral stem cell distribution [18]. Given that PGF intervention after AMI results in an increase in specific gene transcripts, PGF is regarded as a prognostic marker of acute coronary syndrome [19]. To evaluate the cardioprotective potential of PGF, we determined the effect of PGF, injected into the infarction zone of the myocardium either as a nude molecule or in a form of nanoparticles, on the cardiac function after AMI in a rat model in this study. First, we found that both PGF and PGF-PLGA $\mathrm{NPs}_{\mathrm{s}}$ significantly increased LVEF, reduced scar tissue formation, thus leading to recovery of cardiac function, in animals after AMI induction. However, the improvement in all these variables was more significant for PGF-PLGA $\mathrm{NPs}_{\mathrm{s}}$ than PGF.

In order to investigate the mechanism underlying the cardioprotective activity of PGF, we analyzed changes in protein contents of MMPs (MT1-MMP, MMP-2) and its inhibitor (TIMP-2) in infarcted area. The protein content of TIMP-2 at the baseline level was high, but it was reduced after-AMI. PGF intervention was effective to attenuate the reduction. In contrast, an opposite pattern of changes was observed for MT1-MMP and MMP-2 proteins. MMPs belong to a family of $\mathrm{Zn}^{2+-}$ dependent enzymes known to cleave ECM proteins under normal and pathological conditions [20,21]. TIMP-2 may promote

Table 1 Cardiac function parameters assessed by echocardiography

\begin{tabular}{lllll}
\hline & Parameters & & & \\
\cline { 2 - 4 } Groups & LVEF (\%) & LVFS (\%) & LVAW (mm) & Heart rate \\
\hline Sham & $81.28 \pm 3.70$ & $51.78 \pm 11.26$ & $3.23 \pm 0.60$ & $478 \pm 47$ \\
AMl & $21.03 \pm 6.36^{\#}$ & $8.74 \pm 4.08^{\#}$ & $0.87 \pm 0.14^{\#}$ & $497 \pm 52$ \\
PGF & $27.71 \pm 4.59^{\#} \&$ & $16.29 \pm 6.91^{\#} \&$ & $1.28 \pm 0.28^{\#} \&$ & $462 \pm 61$ \\
PGF-PLGA & $26.63 \pm 9.27^{\#} \&$ * & $1.35 \pm 0.38^{\#} \&$ & $481 \pm 59$ \\
\hline
\end{tabular}

AMI acute myocardial infarction; LVEF left ventricular ejection fraction; LVFS left ventricular fraction shortening; LVAW Left ventricular anterior wall. " $\mathrm{p}<0.05$, compared with sham group; \& $\mathrm{p}<0.05$, compared with AMI group; $\aleph_{p}<0.05$, compared with PGF group. 


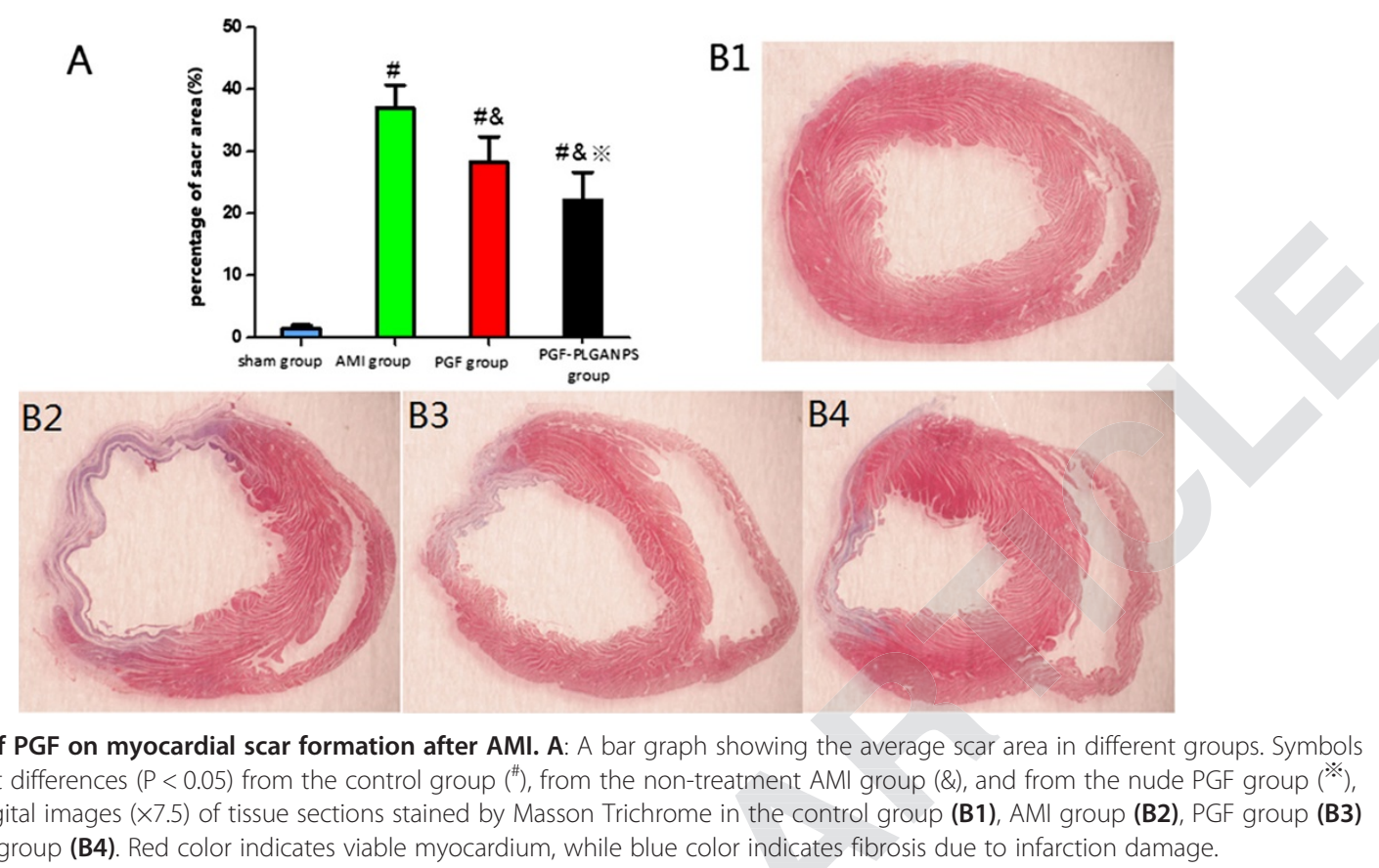

the conversion from pro-MMP-2 to mature MMP-2, acting as a key protein regulating ECM degradation after AMI [22]. Under exogenous PGF intervention, a compensatory increase in TIMP-2 and MT1-MMP expression occurs, leading to an increased production and/or activation of pro-MMP-2 through MT1-MMP [23]. In the process of ECM degradation, MT1-MMP and MMP-2 exert synergistic effects; type I collagen is initially degraded by MT1MMP and the product subsequently degraded by MMP-2 $[23,24]$. Our observations in this study are consistent with the established cascade of ECM degradation in the infarcted myocardial zone after AMI. We also observed myocardial necrosis and collagen, followed by gradual scarring. A large area of blue scar tissues was detected in AMI group, followed by a decrease in TIMP-2 protein content in the myocardium. Following PGF intervention, both TIMP-2 and MMP-2 increased in protein content, resulting in accelerated collagen degradation, in the scar tissue in the MI zone, facilitating the implantation of the new-born cardiomyocytes. TIMP-2 is a natural inhibitor of both MT1-MMP and MMP-2. TIMP-2 deficiency completely abrogates MMP-2 activation but markedly increases activities of various collagenases, particularly MT1-MMP, after MI [5]. Based on our immunohistochemical and Western blotting analysis results, we suggest that post-AMI intervention with PGF may promote the expression recovery of TIMP-2, thus activating MMPs, inhibiting the formation of scar tissues and improving ventricular remodeling.

Nanoparticles are submicron-sized polymeric particles encapsulated with a therapeutic agent within the polymeric matrix or conjugated or absorbed onto their surface
[25], allowing for a controlled release of active agents to the specific site of action at a therapeutically optimal rate [26]. PGF has a short half-life and is not suitable to be exposed in tissues. To obtain a sustained slow-release, multiple types of the nanoparticles have been used to deliver PGF in human body. For instance, in the study of Mohammed et al, they used chitosan-alginate nanoparticle [10], while in this study PLGA was used. We showed that nanoparticles-based delivery enabled persistent and sustained release of PGF, supporting the superiority of nano-materials in drug delivery [27], due to their excellent biocompatibility and biodegradability [28]. It was consistent with the study of Mohammed et al. and others [29,30]. The main advantage of using PLGA as a carrier is that the release profile can be modified by different parameters, such as molecular weight and lactide to glycolide ratio. In this study, a consistently slow release of PGF from nanoparticles was demonstrated during the 15-day period of test. Another advantage of PLGA-based drug delivery is a burst release in the initial period. In this study, an initial burst release of PGF (10\% of total approximately) was observed on the first day. Given significant increases in the expression of pro-inflammatory cytokines and MMPs the first 3 days after AMI [31], the burst release of PGF observed in this study may have important clinical implications.

\section{Conclusion}

PGF may improve the cardiac function after AMI, possibly through a mechanism involving TIMP-2, MT1-MMP, and MMP-2-associated ECM degradation ad tissue remodeling 


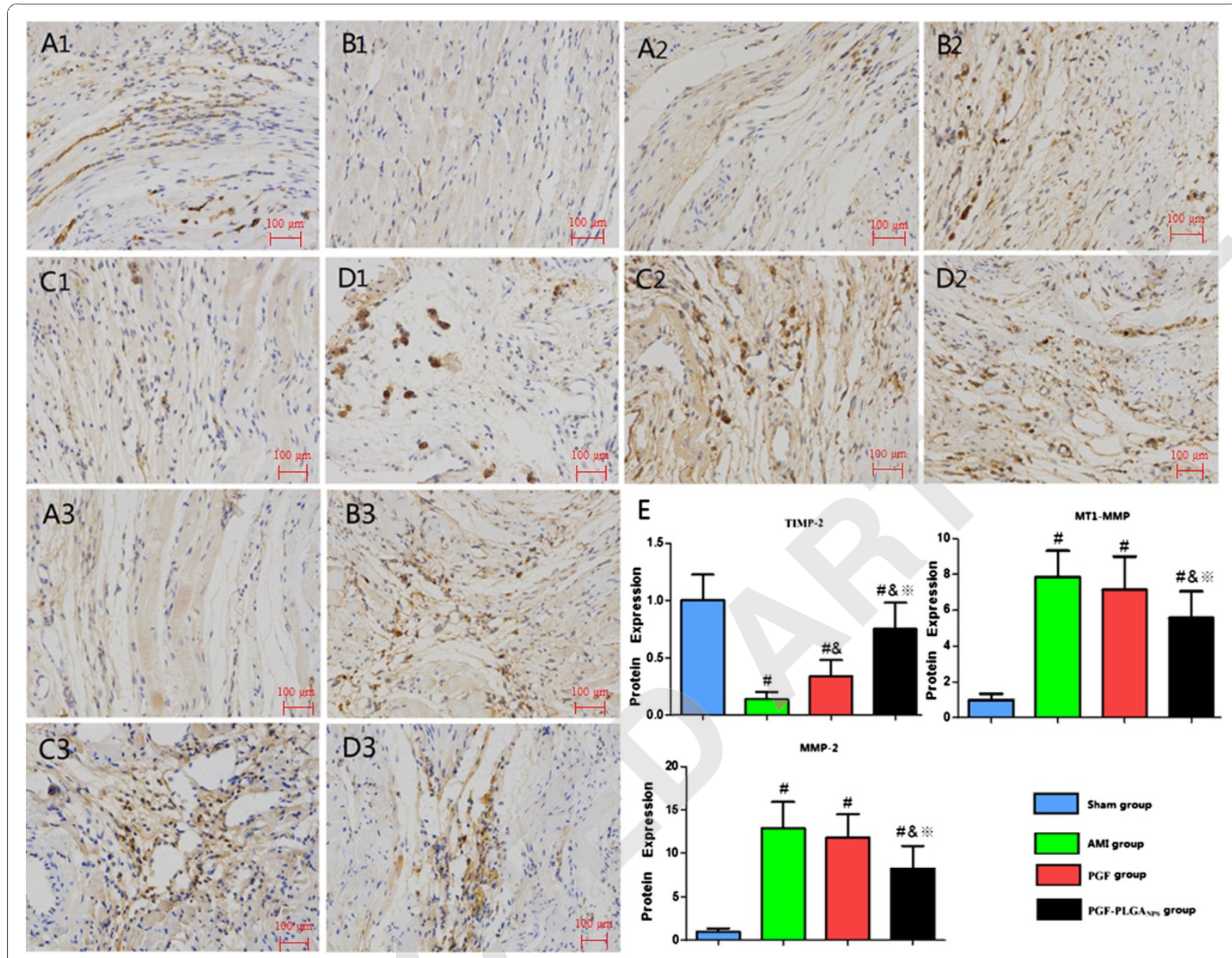

Figure 5 Immunohistochemical signals of TIMP-2 (A1-D1), MT1-MMP (A2-D2), and MMP-2 (A3-D3) (×200) in myocardium in the control group (A), non-treatment AMI group (B), PGF group (C), and PGF-PLGA NPs $_{\text {group }}(\mathrm{D})$. The densitometric data on the corresponding signals in $\mathbf{A}, \mathbf{B}, \mathbf{C}$ and $\mathbf{D}$ are presented as bar graphs in $\mathbf{E}$. Symbols indicate significant differences $(P<0.05)$ from the control group $\left({ }^{\#}\right)$, from the non-treatment AMI group $(\&)$, and from the nude PGF group $(*)$, respectively.

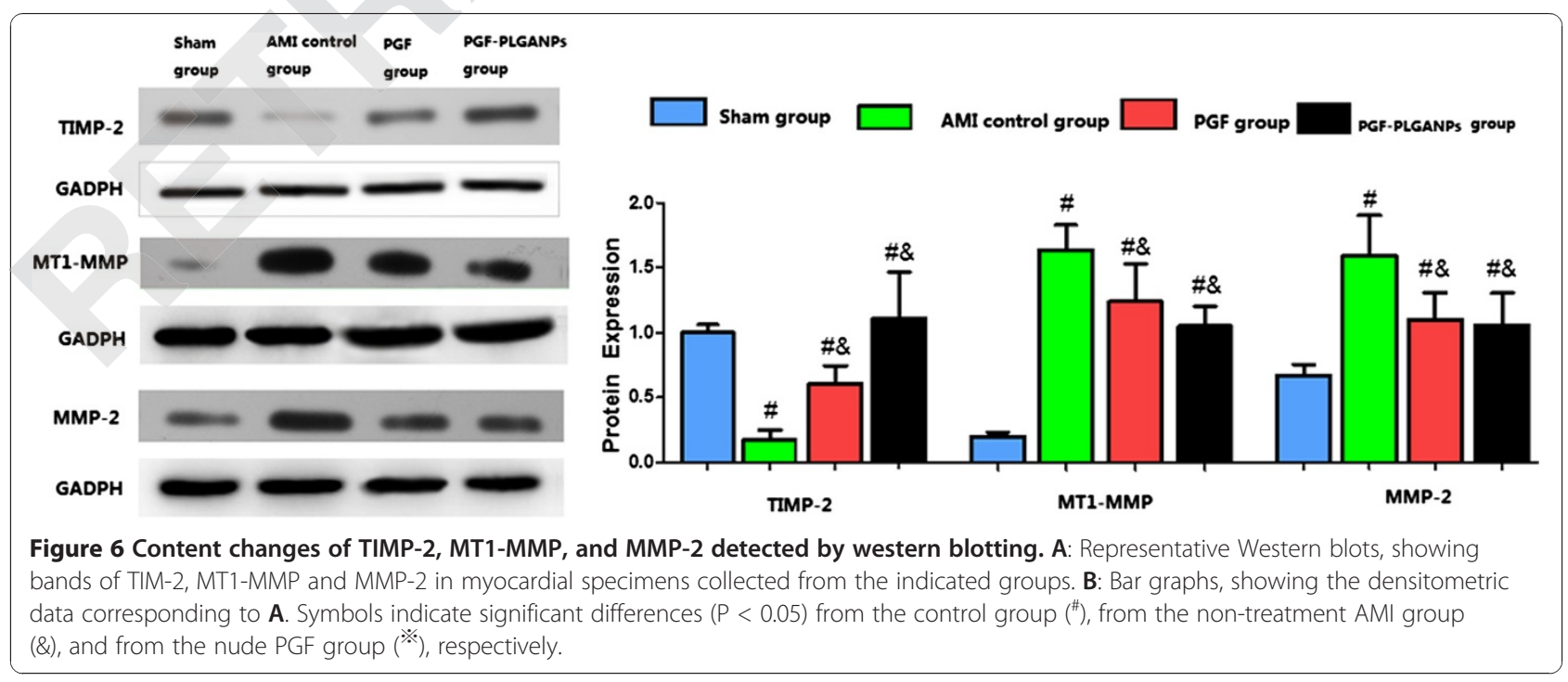


in the myocardium. Due to well-controlled release properties, PGF-PLGA $\mathrm{NPs}_{\mathrm{s}}$ system developed in this study offers a superior approach of myocardial delivery of PGF. Further studies are warranted on the mechanisms underlying the cardioprotective activity of PGF in AMI as well as on the optimized dose and administration time window of PGF$\mathrm{PLGA}_{\mathrm{NPs}}$ as a potential therapeutic approach for AMI.

\section{Competing interests}

The authors declare that they have no competing interests.

\section{Authors' contributions}

ZXL and LLM carried out the molecular genetic studies, participated in the sequence alignment and manuscript preparation. $\mathrm{FH}$ and $\mathrm{JH}$ carried out the immunoassays. WTZ participated in the sequence alignment. CYD, SX and WGL participated in the design of the study and performed the statistical analysis. HSZ conceptualized the study, participated in the study design, oversaw execution of the designed experiments and helped prepare the manuscript. All authors read and approved the final manuscript.

\section{Authors' information}

Zhe-xin Lu and Li-li Mao should be regarded as co-first authors.

\section{Acknowledgements}

We wish to express our warm thanks to Professor Jun Li from Stem Cell Center of Renji Hospital and Dr. Jufang Yao from the Animal Experiment Center of Renji Hospital.

\section{Fundings}

Shanghai Education Commission (11YZ48), Shanghai Education Commission (13ZZ091), Shanghai municipal Scientific \& Technological Commission (12 nm0502100), Shanghai Municipal Bureau of Health P.R. China (No.20114243)

Received: 3 December 2013 Accepted: 4 April 2014 Published: 17 April 2014

\section{References}

1. Chi N-H, Yang M-C, Chung T-W, Chou N-K, Wang S-S: Cardiac repair using chitosan-hyaluronan/silk fibroin patches in a rat heart model with myocardial infarction. Carbohydr Polym 2013, 92(1):591-597.

2. Li T-S, Cheng K, Malliaras K, Smith RR, Zhang Y, Sun B, Matsushita N, Blusztajn A, Terrovitis J, Kusuoka H: Direct comparison of different stem cell types and subpopulations reveals superior paracrine potency and myocardial repair efficacy with cardiosphere-derived cells. J Am Coll Cardiol 2012, 59(10):942-953.

3. Gmeiner M, Zimpfer D, Holfeld J, Seebacher G, Abraham D, Grimm M, Aharinejad S: Improvement of cardiac function in the failing rat heart after transfer of skeletal myoblasts engineered to overexpress placental growth factor. J Thorac Cardiovasc Surg 2011, 141(5):1238-1245.

4. Sutton MGSJ, Sharpe N: Left ventricular remodeling after myocardial infarction pathophysiology and therapy. Circulation 2000, 101(25):2981-2988.

5. Kandalam V, Basu R, Abraham T, Wang X, Soloway PD, Jaworski DM, Oudit GY, Kassiri Z: TIMP2 deficiency accelerates adverse post-myocardial infarction remodeling because of enhanced MT1-MMP activity despite lack of MMP2 activation. Circ Res 2010, 106(4):796-808.

6. Kudo T, Takino T, Miyamori H, Thompson EW, Sato H: Substrate choice of membrane-type 1 matrix metalloproteinase is dictated by tissue inhibitor of metalloproteinase-2 levels. Cancer Sci 2007, 98(4):563-568.

7. Schutz CA, Juillerat-Jeanneret L, Mueller H, Lynch I, Riediker M, NanolmpactNet C: Therapeutic nanoparticles in clinics and under clinical evaluation. Nanomedicine 2013, 8(3):449-467.

8. Danhier F, Ansorena E, Silva JM, Coco R, Le Breton A, Preat V: PLGA-based nanoparticles: an overview of biomedical applications. J Control Release 2012, 161(2):505-522.

9. Li PL, Li CX, Xue YT, Li HH, Liu HB, He XX, Yu GL, Guan HS: An HPLC method for microanalysis and pharmacokinetics of marine sulfated polysaccharide PSS-loaded poly lactic-co-glycolic acid (PLGA) nanoparticles in rat plasma. Mar Drugs 2013, 11(4):1113-1125.
10. Binsalamah ZM, Paul A, Khan AA, Prakash S, Shum-Tim D: Intramyocardial sustained delivery of placental growth factor using nanoparticles as a vehicle for delivery in the rat infarct model. Int I Nanomedicine 2011, 6:2667-2678.

11. Pandey R, Ahmad Z, Sharma S, Khuller G: Nano-encapsulation of azole antifungals: potential applications to improve oral drug delivery. Int $J$ Pharm 2005, 301(1):268-276.

12. Chen G, Nayan M, Duong M, Asenjo J-F, Ge Y, Chiu RC-J, Shum-Tim D: Marrow stromal cells for cell-based therapy: the role of antiinflammatory cytokines in cellular cardiomyoplasty. Ann Thorac Surg 2010, 90(1):190-197.

13. Carnevale D, Lembo G: Placental growth factor and cardiac inflammation. Trends Cardiovasc Med 2012, 22(8):209-212.

14. Iwasaki H, Kawamoto A, Tjwa M, Horii M, Hayashi S, Oyamada A, Matsumoto T, Suehiro S, Carmeliet P, Asahara T: PIGF repairs myocardial ischemia through mechanisms of angiogenesis, cardioprotection and recruitment of myo-angiogenic competent marrow progenitors. PLOS ONE 2011, 6(9):e24872.

15. Takeda Y, Uemura S, Iwama H, Imagawa K-I, Nishida T, Onoue K, Takemoto Y, Soeda T, Okayama S, Somekawa S: Treatment with recombinant placental growth factor (PIGF) enhances both angiogenesis and arteriogenesis and improves survival after myocardial infarction. Circ J 2009, 73(9):1674-1682.

16. Viita $H$, Markkanen J, Eriksson E, Nurminen M, Kinnunen $K$, Babu M, Heikura T, Turpeinen S, Laidinen S, Takalo T: 15-Lipoxygenase-1 prevents vascular endothelial growth factor A-and placental growth factor-induced angiogenic effects in rabbit skeletal muscles via reduction in growth factor mRNA levels, NO bioactivity, and downregulation of VEGF receptor 2 expression. Circ Res 2008, 102(2):177-184.

17. Dayan V, Yannarelli G, Billia F, Filomeno P, Wang X-H, Davies JE, Keating A: Mesenchymal stromal cells mediate a switch to alternatively activated monocytes/macrophages after acute myocardial infarction. Basic Res Cardio/ 2011, 106(6):1299-1310.

18. Cianfarani F, Zambruno G, Brogelli L, Sera F, Lacal PM, Pesce M, Capogrossi MC, Failla CM, Napolitano M, Odorisio T: Placenta growth factor in diabetic wound healing: altered expression and therapeutic potential. Am J Pathol 2006, 169(4):1167-1182.

19. Hattori K, Heissig B, Wu Y, Dias S, Tejada R, Ferris B, Hicklin DJ, Zhu Z, Bohlen $P$, Witte L: Placental growth factor reconstitutes hematopoiesis by recruiting VEGFR1+ stem cells from bone-marrow microenvironment. Nat Med 2002, 8(8):841-849.

20. Raffetto JD, Khalil RA: Matrix metalloproteinases and their inhibitors in vascular remodeling and vascular disease. Biochem Pharmacol 2008, 75(2):346-359.

21. Spinale FG: Myocardial matrix remodeling and the matrix metalloproteinases: influence on cardiac form and function. Physiol Rev 2007, 87(4):1285-1342.

22. Kandalam V, Basu R, Moore L, Fan D, Wang X, Jaworski DM, Oudit GY, Kassiri $Z$ : Lack of tissue inhibitor of metalloproteinases 2 leads to exacerbated left ventricular dysfunction and adverse extracellular matrix remodeling in response to biomechanical StressClinical perspective. Circulation 2011, 124(19):2094-2105.

23. Nishida Y, Miyamori H, Thompson EW, Takino T, Endo Y, Sato H: Activation of matrix metalloproteinase-2 (MMP-2) by membrane type 1 matrix metalloproteinase through an artificial receptor for proMMP-2 generates active MMP-2. Cancer Res 2008, 68(21):9096-9104.

24. Bujak M, Frangogiannis NG: The role of TGF- $\beta$ signaling in myocardial infarction and cardiac remodeling. Cardiovasc Res 2007, 74(2):184-195.

25. Douglas S, Davis S, Illum L: Nanoparticles in drug delivery. Crit Rev Ther Drug Carrier Syst 1987, 3(3):233.

26. Soppimath KS, Aminabhavi TM, Kulkarni AR, Rudzinski WE: Biodegradable polymeric nanoparticles as drug delivery devices. J Control Release 2001, 70(1):1-20.

27. Shriver LP, Koudelka KJ, Manchester M: Viral nanoparticles associate with regions of inflammation and blood brain barrier disruption during CNS infection. J Neuroimmunol 2009, 211(1):66-72.

28. Bala I, Hariharan S, Kumar MR: PLGA nanoparticles in drug delivery: the state of the art. Crit Rev Therap Drug Carrier Syst 2004, 21(5):387-422.

29. Karra N, Nassar T, Ripin AN, Schwob O, Borlak J, Benita S: Antibody conjugated PLGA nanoparticles for targeted delivery of paclitaxel palmitate: efficacy and biofate in a lung cancer mouse model. Small 2013, 9(24):4221-4236. 
30. Kim DH, Martin DC: Sustained release of dexamethasone from hydrophilic matrices using PLGA nanoparticles for neural drug delivery. Biomaterials 2006, 27(15):3031-3037.

31. Pereira MJ, Carvalho IF, Karp JM, Ferreira LS: Sensing the cardiac environment: exploiting cues for regeneration. J Cardiovasc Trans/ Res 2011, 4(5):616-630.

doi:10.1186/1471-2261-14-53

Cite this article as: Lu et al:: Cardioprotective activity of placental growth factor in a rat model of acute myocardial infarction: nanoparticle-based delivery versus direct myocardial injection. BMC Cardiovascular Disorders 2014 14:53.

\section{Submit your next manuscript to BioMed Central and take full advantage of:}

- Convenient online submission

- Thorough peer review

- No space constraints or color figure charges

- Immediate publication on acceptance

- Inclusion in PubMed, CAS, Scopus and Google Scholar

- Research which is freely available for redistribution 